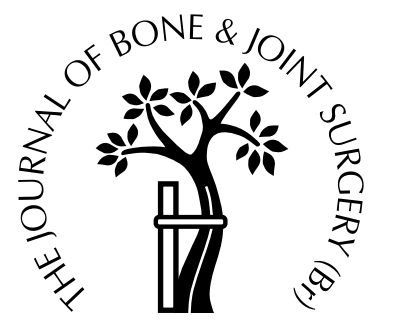

\title{
Apoptosis and expression of stress protein (ORP150, HO1) during development of ischaemic osteonecrosis in the rat
}

\author{
M. Sato, N. Sugano, K. Ohzono, S. Nomura, \\ Y. Kitamura, Y. Tsukamoto, S. Ogawa \\ From Osaka University Medical School, Osaka, Japan
}

$\mathbf{U}$ sing in situ hybridisation and the terminal deoxynucleotidyl transferase-mediated biotin-dUTP nick end-labelling (TUNEL) reaction in rats with osteonecrosis of the femoral head we have studied the effect of ischaemia on the gene expression of the stress proteins oxygen-regulated protein 150 (ORP150) and haemoxygenase 1 (HO1) and the death mechanism of the cells involved in osteonecrosis. Both ORP150 and HO1 have been reported to have important roles in the successful adaptation to oxygen deprivation.

ORP150 and HO1 mRNA expression was induced by ischaemia in osteoblasts and osteocytes. In proliferative chondrocytes, these signals were detected constitutively. During the development of ischaemic osteonecrosis, the mechanism of cell death was apoptosis as indicated by DNA fragmentation and the presence of apoptotic bodies in osteocytes, chondrocytes and bone-marrow cells. After the initial ischaemic event, expression of ORP150 and HO1 mRNA, the TUNEL-positive reaction and empty lacunae were found sequentially. These findings were exclusive and may be considered to be markers for each stage in the development of osteonecrosis.

J Bone Joint Surg [Br] 2001;83-B:751-9.

Received 1 December 1999; Accepted after revision 9 June, 2000

M. Sato, MD, DMSc, Orthopaedic Fellow

N. Sugano, MD, DMSc, Assistant Professor

K. Ohzono, MD, DMSc, Associate Professor

Department of Orthopaedic Surgery

S. Nomura, PhD, Associate Professor

Y. Kitamura, MD, DMSc, Professor and Chairman

Department of Pathology

Osaka University Medical School, 2-2, Yamadaoka, Suita, Osaka 565-0871, Japan.

Y. Tsukamoto, MD, DMSc, Assistant Professor

Department of Pathology, National Cardiovascular Centre, 5-7-1 Fujishiro-dai, Suita 565-8565, Japan.

S. Ogawa, MD, DMSc, Professor and Chairman

Department of Neuroanatomy, Kanazawa University School of Medicine, 13-1 Takara-machi, Kanazawa, Ishikawa 920-8640, Japan.

Correspondence should be sent to Dr N. Sugano.

(C)2001 British Editorial Society of Bone and Joint Surgery 0301-620X/01/510801\$2.00
Traumatic and non-traumatic osteonecrosis of the femoral head is assumed to have an ischaemic origin although the pathomechanism of non-traumatic osteonecrosis is still unclear. Even with variable aetiological factors, the pathology is quite similar. ${ }^{1}$ After the initial ischaemic events, death of bone and marrow elements occurs while the articular cartilage is intact. Although osteonecrosis is often diagnosed histologically under light microscopy by the presence of empty lacunae, the precise cellular and molecular basis of these changes in bone has not been clarified and elucidation of this may help in the understanding of the pathomechanism of non-traumatic osteonecrosis.

It has been reported that deprivation of environmental oxygen induces expression of a set of stress proteins ${ }^{2,3}$ which are believed to have an important role in protecting cellular biosynthetic activities under stressful circumstances. Recently, a 150-kD polypeptide termed oxygen-regulated protein (ORP150) has been isolated and cloned and was found to be induced selectively in astrocytes by hypoxia. $^{3}$ In this respect ORP150 differs from haemoxygenase 1 (HO1) which shows enhanced expression in response to hypoxia, as well as other stimuli such as heat shock, heavy metals, endotoxin and inflammatory cytokines. ${ }^{4,5}$ ORP150 plays a pivotal role in the cytoprotective cellular mechanisms triggered by oxygen deprivation, one of which is the inhibition of apoptosis. ${ }^{6}$

We have used in situ hybridisation and the terminal deoxynucleotidyl transferase-mediated biotin-dUTP nick end-labelling (TUNEL) reaction, to demonstrate the spatial localisation and temporal expression of mRNAs for ORP150 and HO1, and TUNEL-positive cells during the development of ischaemic osteonecrosis.

\section{Materials and Methods}

The experimental procedures in this study were undertaken in compliance with the USA guidelines for the care and use of animals. We used 42 male Sprague-Dawley rats, 11 weeks old and weighing about $400 \mathrm{~g}$ (Charles River Japan, Tokyo, Japan). The operation was performed under general anaesthesia with ketamine $(90 \mathrm{mg} / \mathrm{kg})$ and xylazine $(10 \mathrm{mg} /$ $\mathrm{kg}$ ). The left femoral head of each rat was made surgically avascular by the following technique. A skin incision was made over the greater trochanter and the hip capsule 


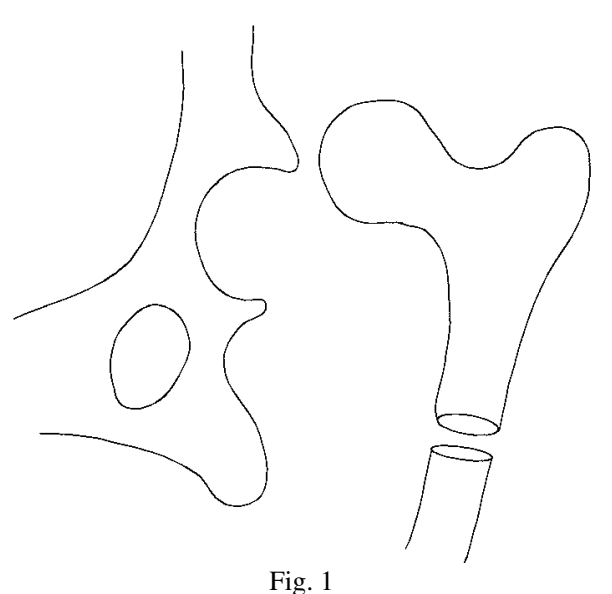

Diagram of the operation. The hip was dislocated and the transverse osteotomy performed.

Table I. The number of rats at each stage of the experiment

\begin{tabular}{lllllll}
\hline & \multicolumn{7}{l}{ Hours after operation } \\
\cline { 2 - 7 } & $\mathbf{1 . 5}$ & $\mathbf{3}$ & $\mathbf{6}$ & $\mathbf{1 2}$ & $\mathbf{2 4}$ & $\mathbf{9 6}$ \\
\hline Number of rats & 5 & 7 & 7 & 9 & 9 & 5 \\
\hline
\end{tabular}

exposed. This was then excised circumferentially, the round ligament divided and the hip dislocated. The soft-tissue attachments were then stripped from the femoral neck and intertrochanteric area. In order to disrupt the vessels which feed the femoral head via the vascular network from the diaphysis, a transverse osteotomy was performed $20 \mathrm{~mm}$ distal to the femoral head (Fig. 1). The free proximal segment of the femur was reduced to the original position and the soft tissue around the femur was repaired. No internal or external fixation was used. The contralateral femur was used as a control. The animals were killed at various postoperative stages for histological examination and DNA extraction (Table I).

For in situ hybridisation and the TUNEL reaction, the animals were perfused under general anaesthesia and both femora were excised. The bones were fixed and decalcified and histological sections were prepared as described pre- viously. ${ }^{7}$ For DNA extraction, the proximal segment of the femur was excised and homogenised.

We used the following complementary DNA (cDNA) clones as hybridisation probes: rat ORP150 cDNA containing a $0.23 \mathrm{~kb}$ (151 to 381) fragment and human HO1 cDNA containing a $0.64 \mathrm{~kb}$ (377 to 1014) fragment. These cDNAs were subcloned into the EcoRV site of pBluescript KS(Stratagene, La Jolla, California).

In situ hybridisation. We used the same technique as has been described elsewhere. ${ }^{77}$ Digoxigenin-labelled singlestrand RNA probes were prepared for hybridisation using a DIG RNA labelling kit (Boehringer Mannheim Biochemica, Mannheim, Germany) according to the manufacturer's instructions. Hybridisation of ORP150 and HO1 mRNA was performed at $50^{\circ} \mathrm{C}$ for 16 hours, and the signals detected using a nucleic acid detection kit (Boehringer Mannheim Biochemica). Control experiments included: 1) hybridisation with the sense probes; 2) RNase treatment before hybridisation; and 3) use of neither the antisense RNA probe nor anti-digoxigenin antibody. All three gave no detectable signal.

End labelling of tissue sections using a terminal deoxynucleotidyl transferase (TUNEL). This was performed using ApopTag (Oncor, Gaithersberg, Maryland) according to the manufacturer's instructions. Controls for the study included samples which had not been treated with the antibody and tissue that had not been treated with the terminal transferase.

DNA fragmentation analysis by agarose gel electrophoresis. Additional animals subjected to 12 hours or 24 hours of ischaemia were used for the gel electrophoresis assay. DNA was extracted from the femora using standard procedures. ${ }^{8}$ The DNA was then electrophoresed on a $1.5 \%$ agarose gel which was reacted with ethidium bromide and the DNA visualised by an ultraviolet transilluminator.

\section{$\underline{\text { Results }}$}

All animals survived the operation. The results are summarised in Table II.

Normal femoral head. The centre of ossification of the

Table II. Localisation of the TUNEL-positive reaction and mRNA encoding HO1 and ORP150 during development of ischaemic osteonecrosis

\begin{tabular}{|c|c|c|c|c|c|c|c|c|c|c|c|c|c|c|c|c|c|c|}
\hline & \multicolumn{6}{|c|}{ Epiphysis } & \multicolumn{6}{|c|}{ Growth plate } & \multicolumn{6}{|c|}{ Metaphysis } \\
\hline & \multicolumn{3}{|l|}{ OB* } & \multicolumn{3}{|l|}{ OC* } & \multicolumn{3}{|l|}{$\mathbf{P C}^{*}$} & \multicolumn{3}{|l|}{ HC $^{*}$} & \multicolumn{3}{|l|}{$\overline{\mathbf{O B}}$} & \multicolumn{3}{|l|}{ OC } \\
\hline & $\overline{\mathrm{HO1}}$ & ORP† & $\overline{\mathbf{T N} \dagger}$ & HO1 & ORP & TN & $\overline{\mathrm{HO1}}$ & ORP & TN & $\overline{\mathrm{HO1}}$ & ORP & TN & $\overline{\mathrm{HO1}}$ & ORP & TN & $\overline{\mathrm{HO1}}$ & ORP & $\overline{T N}$ \\
\hline Normal & - & - & - & - & - & - & + & + & - & - & - & + & - & - & - & - & - & - \\
\hline $1.5 \mathrm{hrs}$ & - & - & - & - & - & - & + & + & - & - & - & + & - & - & - & - & - & - \\
\hline $3 \mathrm{hrs}$ & + & + & - & + & + & - & + & + & - & - & - & + & + & + & - & + & + & - \\
\hline $6 \mathrm{hrs}$ & \multirow{3}{*}{\multicolumn{3}{|c|}{ Died out }} & + & + & - & + & + & - & - & - & + & + & + & - & + & + & - \\
\hline $12 \mathrm{hrs}$ & & & & - & - & + & + & + & - & - & - & + & \multirow{2}{*}{\multicolumn{3}{|c|}{ Died out }} & - & - & + \\
\hline $24 \mathrm{hrs}$ & & & & - & - & + & - & - & + & - & - & + & & & & - & - & + \\
\hline $96 \mathrm{hrs}$ & & & & \multicolumn{3}{|c|}{ Empty lacunae } & - & - & + & - & - & + & & & & \multicolumn{3}{|c|}{ Empty lacunae } \\
\hline
\end{tabular}

* OB, osteoblasts; OC, osteocytes; PC, proliferative chondrocytes; $\mathrm{HC}$, terminally differentiated hypertrophic chondrocytes

$\dagger$ ORP, ORP150; TN, TUNEL-positive reaction 

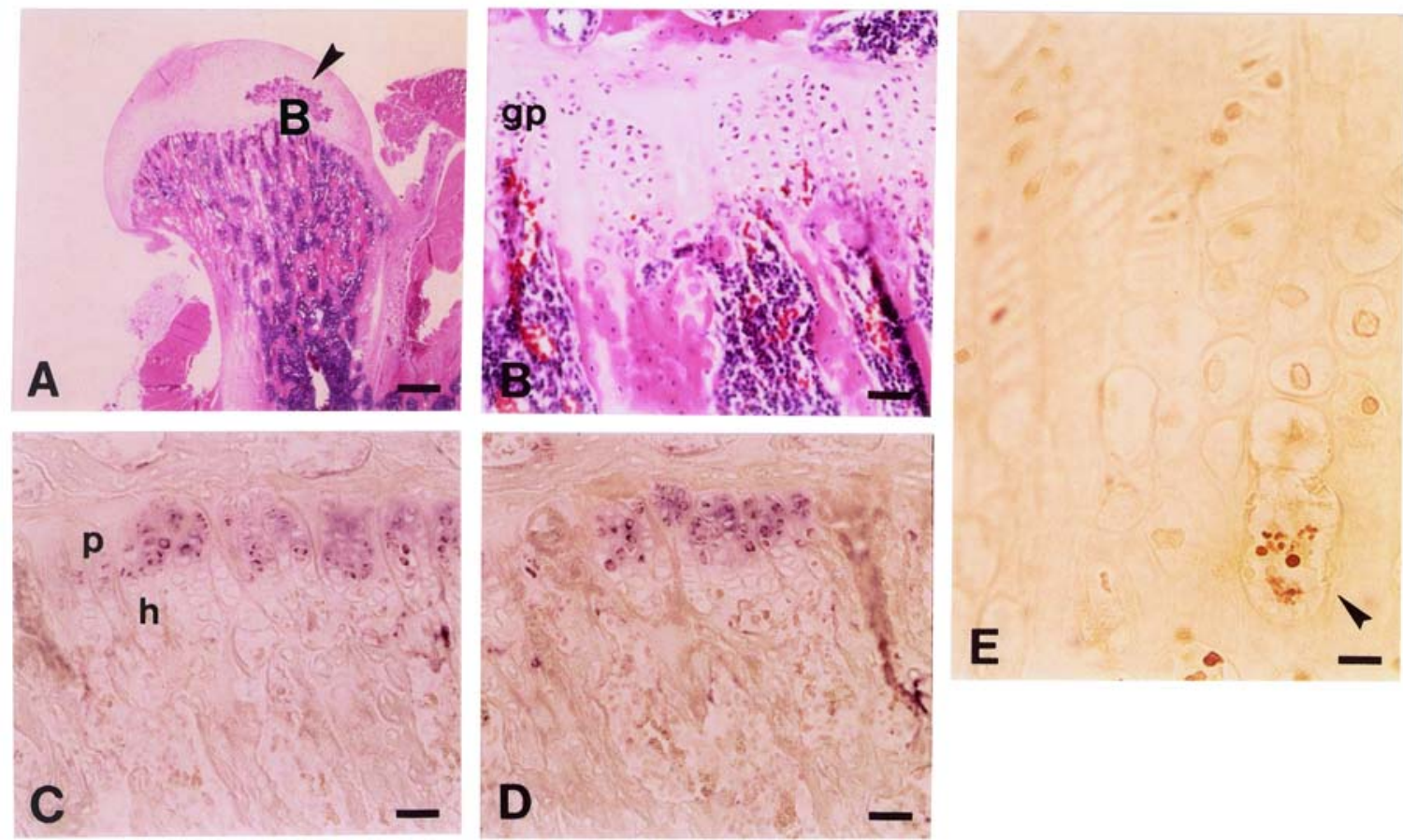

Fig. 2

Photomicrographs showing the histological changes, the TUNEL reaction and localisation of HO1 and ORP150 mRNA in a longitudinal section of the normal femoral head. Figure $2 \mathrm{~A}$ - The ossification centre (arrowhead) is seen in the epiphysis (bar $=710 \mu \mathrm{m}$; haematoxylin and eosin). Figure 2B - A high-power view of the growth plate (area B in Figure 2A; gp, growth plate; bar $=45 \mu \mathrm{m}$; haematoxylin and eosin). Figure $2 \mathrm{C}$ - The HO1 mRNA signal in proliferative chondrocytes (p, proliferative chondrocytes; $h$, hypertrophic chondrocytes; bar $=45 \mu \mathrm{m})$. Figure 2D - The ORP150 mRNA signal in cell types similar to HO1 mRNA-expressing cells $($ bar $=45 \mu \mathrm{m})$. Figure $2 \mathrm{E}-$ Apoptotic bodies are seen in a terminal differentiated hypertrophic chondrocyte (arrowhead) $(\mathrm{bar}=11 \mu \mathrm{m})$.

epiphysis appeared in the femoral head (Figs 2A and 2B). In situ hybridisation demonstrated the localisation of mRNA for HO1 and ORP150. HO1 mRNA was detected in proliferative chondrocytes of the growth plate alone and not in terminally differentiated hypertrophic chondrocytes (Fig. 2C). Osteoblasts and osteocytes expressed no signal. ORP150 mRNA showed a similar pattern of localisation to HO1 mRNA (Fig. 2D). After TUNEL staining, a positive reaction and formation of apoptotic bodies were seen in terminally differentiated hypertrophic chondrocytes (Fig. 2E).

No histological changes or alterations in expression compared with the controls (data not shown) were seen 1.5 hours after operation.

After three hours there was no change histologically (Figs $3 \mathrm{~A}$ and 3B), but expression of HO1 and ORP150 mRNA was induced. Figure $3 \mathrm{C}$ shows active and plump osteoblasts at the bone surface in the ossification centre. HO1 mRNA was expressed in osteoblasts and osteocytes in addition to proliferative chondrocytes (Figs 3D to 3F). ORP150 mRNA was clearly detected in osteoblasts and osteocytes in addition to proliferative chondrocytes (Figs $3 \mathrm{G}$ to $3 \mathrm{I})$.

Six hours after operation a histological change was observed. The active and cuboidal osteoblasts in the epiphysis, which had been observed at three hours, had died or flattened (Figs 4A to 4C), but those in the metaphysis retained their active form and expressed HO1 and ORP150 mRNA (Figs 4D, 4E, 4G and 4H). Osteocytes in the ossification centre expressed ORP150 (Figs 4G and 4I), but not HO1 (Fig. 4D). Little TUNEL staining was seen at this time (Fig. 4F).

Twelve hours after operation the active osteoblasts in the epiphysis and metaphysis had disappeared. Osteocytes showed no histological change and empty osteocytic lacunae were not detected (Fig. 5A). HO1 (Fig. 5B) and ORP150 (Fig. 5C) mRNA were seen in proliferative chondrocytes alone. After TUNEL staining, a positive reaction was observed in osteocytes and bone-marrow cells but not in proliferative chondrocytes which expressed $\mathrm{HO} 1$ and/or ORP150 (Fig. 5D).

After 24 hours the histological features were similar to those at 12 hours (Fig. 6A). No signal of HO1 or ORP150 was detected (data not shown). After TUNEL staining, a positive reaction was observed in various cell types including osteocytes, chondrocytes and bone-marrow cells (Fig. $6 \mathrm{~B})$ and the formation of apoptotic bodies was observed in osteocytes and bone-marrow cells (Figs 6C and 6D).

After 96 hours some of the osteocytic lacunae were empty, which was thought to be a traditional marker of osteonecrosis (Fig. 7A). No signal of HO1 or ORP150 was detected (data not shown). TUNEL-positive cells were 

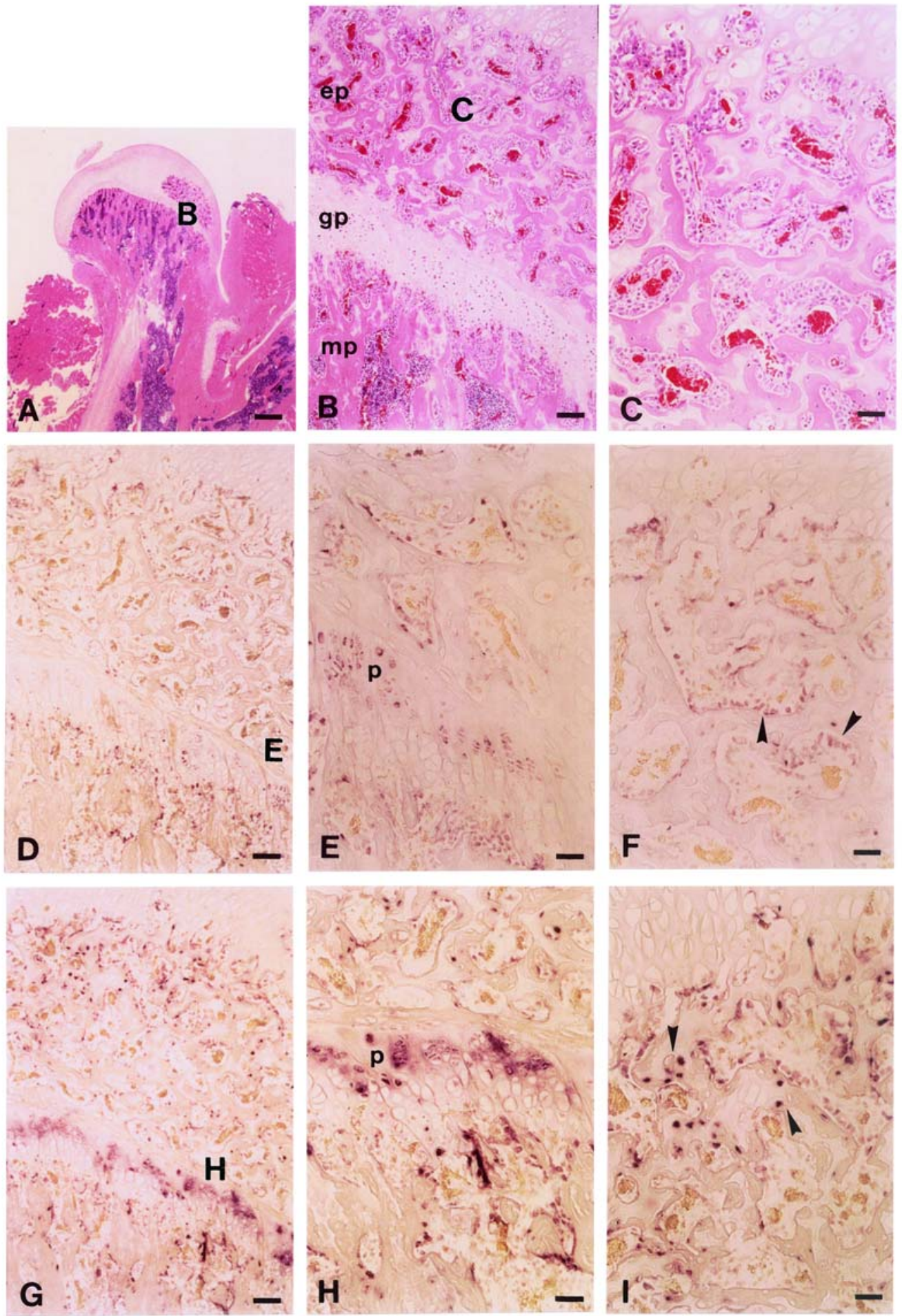

Fig. 3

Photomicrographs of the histological changes and localisation of HO1 and ORP150 mRNA in a longitudinal section of the femoral head after three hours of ischaemia. Figure $3 \mathrm{~A}$ - The growth plate $(\mathrm{bar}=710 \mu \mathrm{m}$, haematoxylin and eosin). Figure $3 \mathrm{~B}$ - High-power view of the growth plate (area B in Figure 3A; ep, epiphysis; gp, growth plate; mp, metaphysis; bar $=90 \mu \mathrm{m}$ ). Figure $3 \mathrm{C}-$ High-power view of the ossification centre (region $\mathrm{C}$ in Figure 3B). There are active and plump osteoblasts at the bone surface $(\mathrm{bar}=45 \mu \mathrm{m})$. Figure 3D - The HO1 mRNA signal in osteoblasts, osteocytes and proliferative chondrocytes $(\mathrm{bar}=90 \mu \mathrm{m})$. Figure $3 \mathrm{E}-$ High-power view of the growth plate (area E in Figure 3D) showing the HO1 mRNA signal in proliferative chondrocytes (p, proliferative chondrocyte; bar $=45 \mu \mathrm{m}$ ). Figure $3 \mathrm{~F}-$ The HO1 mRNA signal in osteoblasts (arrowhead) and osteocytes $(\mathrm{bar}=45 \mu \mathrm{m})$. Figure 3G - The ORP150 mRNA signal in osteoblasts, osteocytes and proliferative chondrocytes $(\mathrm{bar}=90 \mu \mathrm{m})$. Figure $3 \mathrm{H}-\mathrm{High}-$ power view of the growth plate (area H in Figure 3G) showing the ORP150 mRNA signal in proliferative chondrocytes (p, proliferative chondrocyte; bar $=45 \mu \mathrm{m}$ ). Figure 3I - The ORP150 mRNA signal in osteoblasts and osteocytes (arrowhead) of the ossification centre $(\mathrm{bar}=45 \mu \mathrm{m})$. 

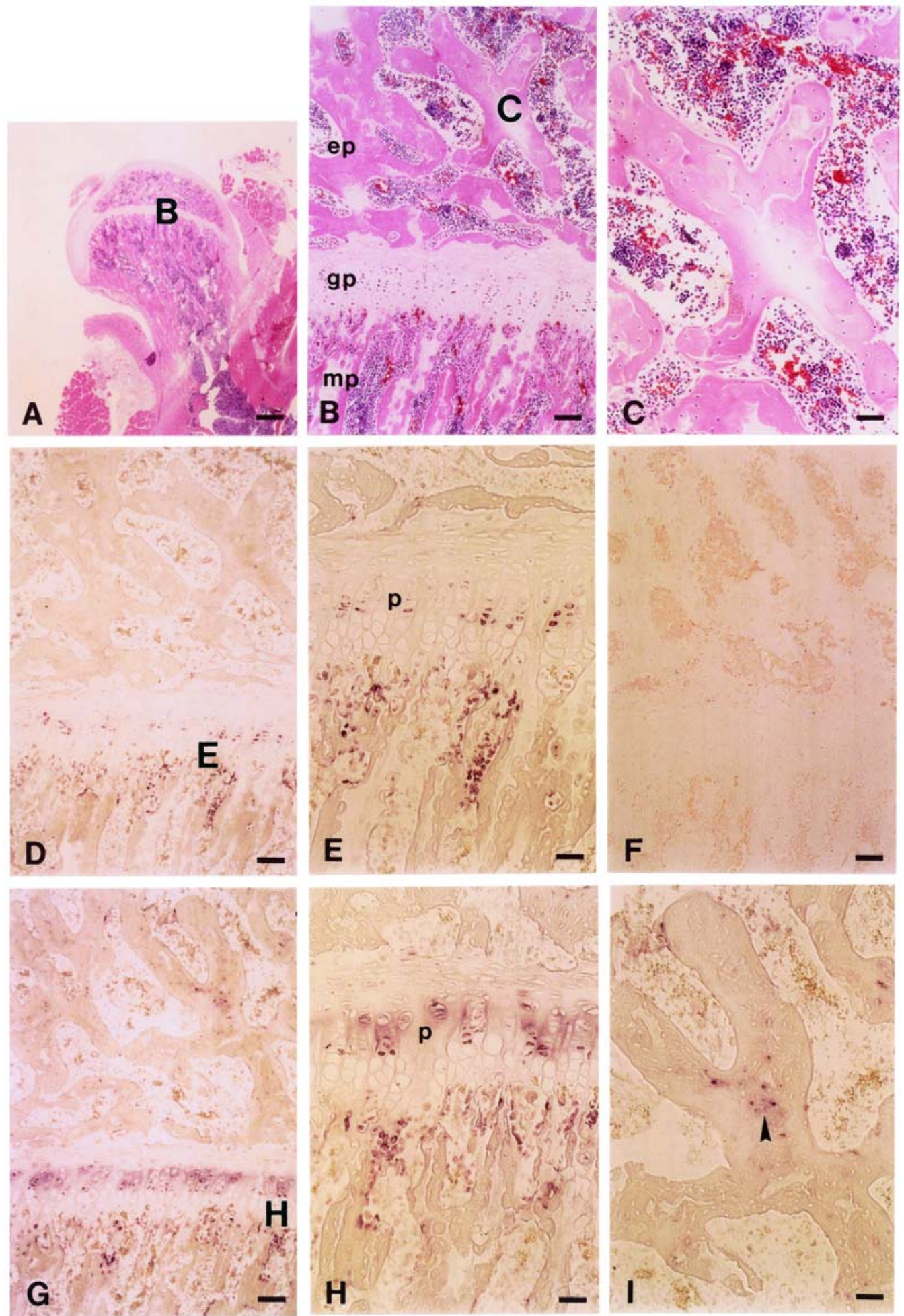

Fig. 4

Photomicrographs showing the histological changes, the TUNEL reaction and localisation of HO1 and ORP150 mRNA in a longitudinal section of the femoral head after six hours of ischaemia. Figure $4 \mathrm{~A}-$ The growth plate (bar $=710 \mu \mathrm{m}$; haematoxylin and eosin). Figure $4 \mathrm{~B}-\mathrm{High}$-power view of the growth plate (area B in Figure 4A; ep, epiphysis; gp, growth plate; mp, metaphysis; bar $=90 \mu \mathrm{m}$ ). Figure 4C - High-power view of the ossification centre (area C in Figure 4B) showing that the osteoblasts have disappeared or flattened (bar $=45 \mu \mathrm{m})$. Figure $4 \mathrm{D}-$ The HO1 mRNA signal in osteoblasts in the metaphysis and proliferative chondrocytes in the growth plate $(\mathrm{bar}=90 \mu \mathrm{m})$. Figure $4 \mathrm{E}-$ High-power view of the growth plate (area E in Figure 4D) showing the HO1 mRNA signal in proliferative chondrocytes ( $\mathrm{p}$, proliferative chondrocyte; bar $=45 \mu \mathrm{m}$ ). Figure $4 \mathrm{~F}-$ The TUNEL reaction showing few positive cells $(\mathrm{bar}=45 \mu \mathrm{m})$. Figure $4 \mathrm{G}$ - The ORP150 mRNA signal in osteoblasts in the metaphysis, osteocytes and proliferative chondrocytes $(\mathrm{bar}=45 \mu \mathrm{m})$. Figure $4 \mathrm{H}-$ High-power view of the growth plate (area $\mathrm{H}$ in Figure $4 \mathrm{G}$ showing the ORP150 mRNA signal in proliferative chondrocytes in the growth plate and osteoblasts in the metaphysis (p, proliferative chondrocyte; bar $=90 \mu \mathrm{m}$ ). Figure $4 \mathrm{I}-\mathrm{The}$ ORP150 mRNA signal in osteocytes (arrowhead) of the ossification centre (bar $=45 \mu \mathrm{m})$. 

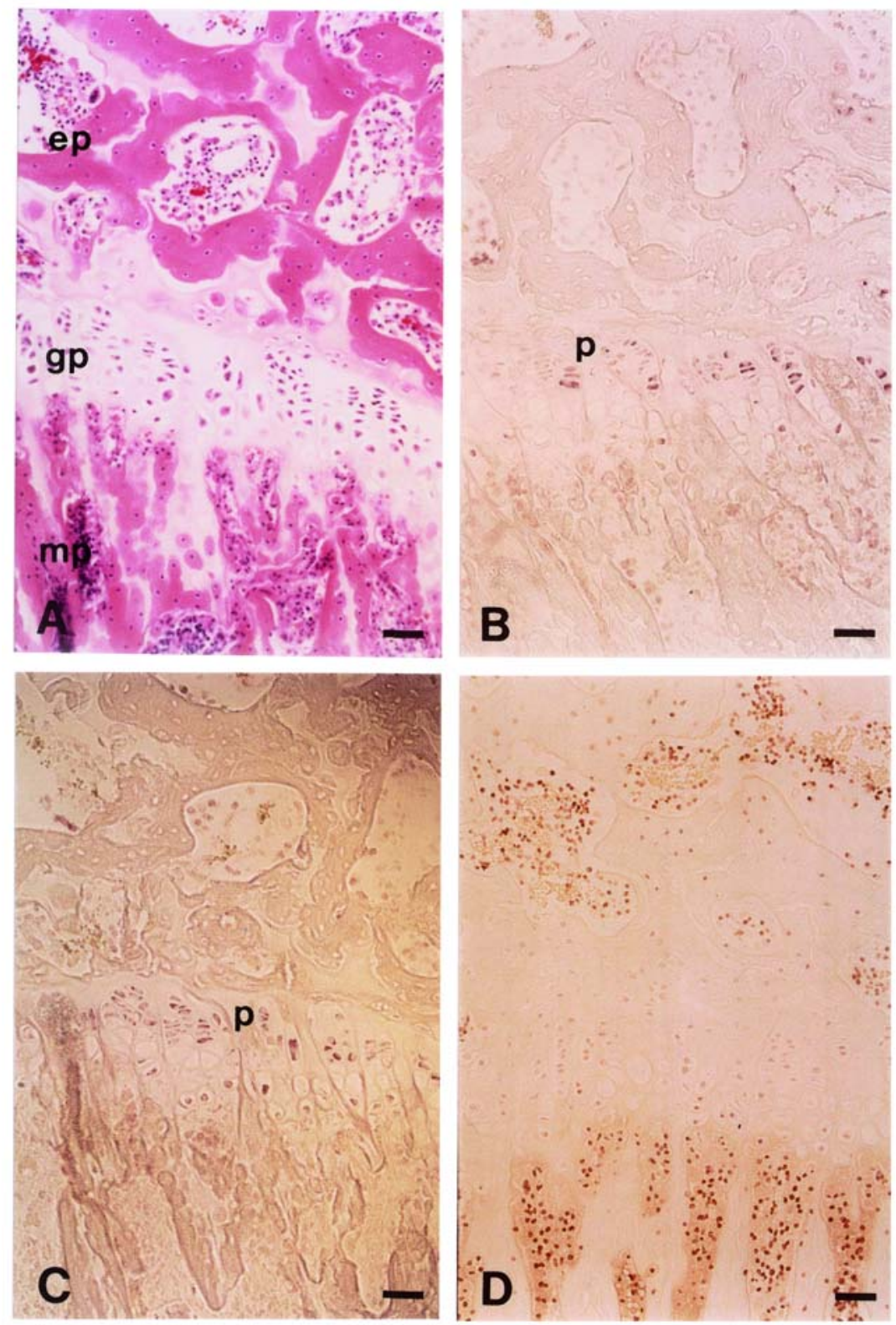

Fig. 5

Photomicrographs of the histological changes, the TUNEL reaction and localisation of HO1 and ORP150 mRNA in a longitudinal section of the femoral head after 12 hours of ischaemia. Figure $5 \mathrm{~A}$ - The osteoblasts in the epiphysis and metaphysis have disappeared (ep, epiphysis; gp, growth plate; mp, metaphysis; bar $=90 \mu \mathrm{m}$; haematoxylin and eosin). Figure $5 \mathrm{~B}-$ The HO1 mRNA signal in proliferative chondrocytes in the growth plate but not in osteocytes (p, proliferative chondrocyte; $\mathrm{bar}=90 \mu \mathrm{m})$. Figure $5 \mathrm{C}-$ The ORP150 mRNA signal in proliferative chondrocytes in the growth plate but not in osteocytes ( $\mathrm{p}$, proliferative chondrocyte; bar $=90 \mu \mathrm{m})$. Figure 4D - The TUNEL reaction in osteocytes and bone-marrow cells, bar $=90 \mu \mathrm{m})$.

decreased in comparison to 24 hours after operation (Fig. 7B) and the formation of apoptotic bodies was observed in chondrocytes (Fig. 7C).

Agarose gel electrophoresis. DNA extracted from the femora subjected to ischaemic insults for 12 and 24 hours showed the formation of DNA fragments in integer multiples of 180 to 200 base pairs, a characteristic 'ladder' pattern (data not shown).

\section{Discussion}

To date, there has been no study in vivo of the influence of ischaemia on the gene expression or death mechanism of cells involved in osteonecrosis. Using in situ hybridisation in a rat model we have provided new insight into the cellular and molecular mechanism of ischaemic osteonecrosis. The mechanism of the cell death initiated by an ischae- 

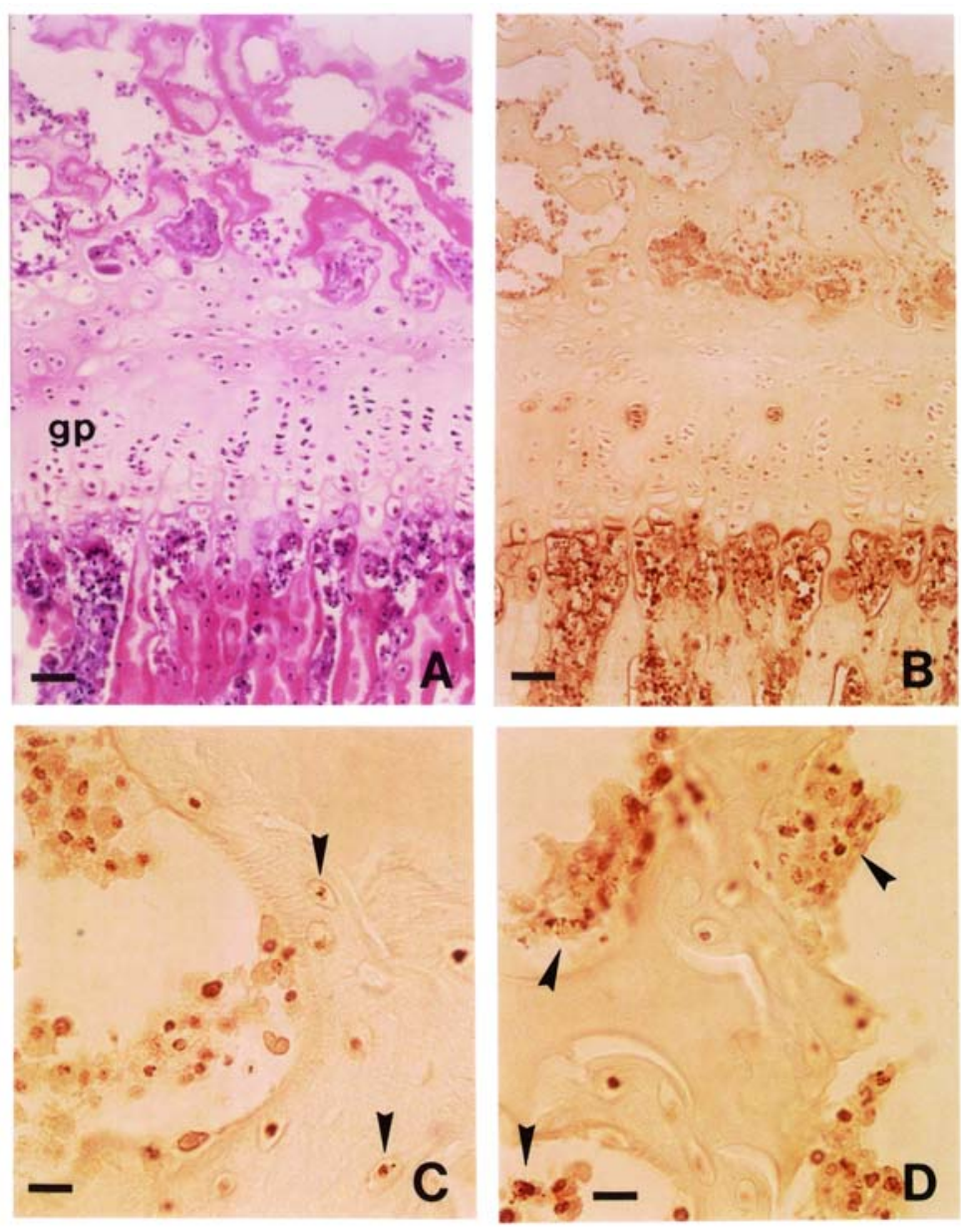

Fig. 6

Photomicrographs of the histological changes and the TUNEL reaction in a longitudinal section of the femoral head after 24 hours of ischaemia. Figure 6A - The growth plate (gp, growth plate; bar $=90 \mu \mathrm{m}$; haematoxylin and eosin). Figure 6B - The TUNEL reaction in osteocytes, chondrocytes and bone-marrow cells $($ bar $=90 \mu \mathrm{m})$. Figure $6 \mathrm{C}-$ The TUNEL reaction showing apoptotic bodies in osteocytes (arrowheads) $(\mathrm{bar}=22 \mu \mathrm{m})$. Figure $6 \mathrm{D}-$ The TUNEL reaction showing apoptotic bodies in bone-marrow cells (arrowheads) $(\mathrm{bar}=22 \mu \mathrm{m})$.
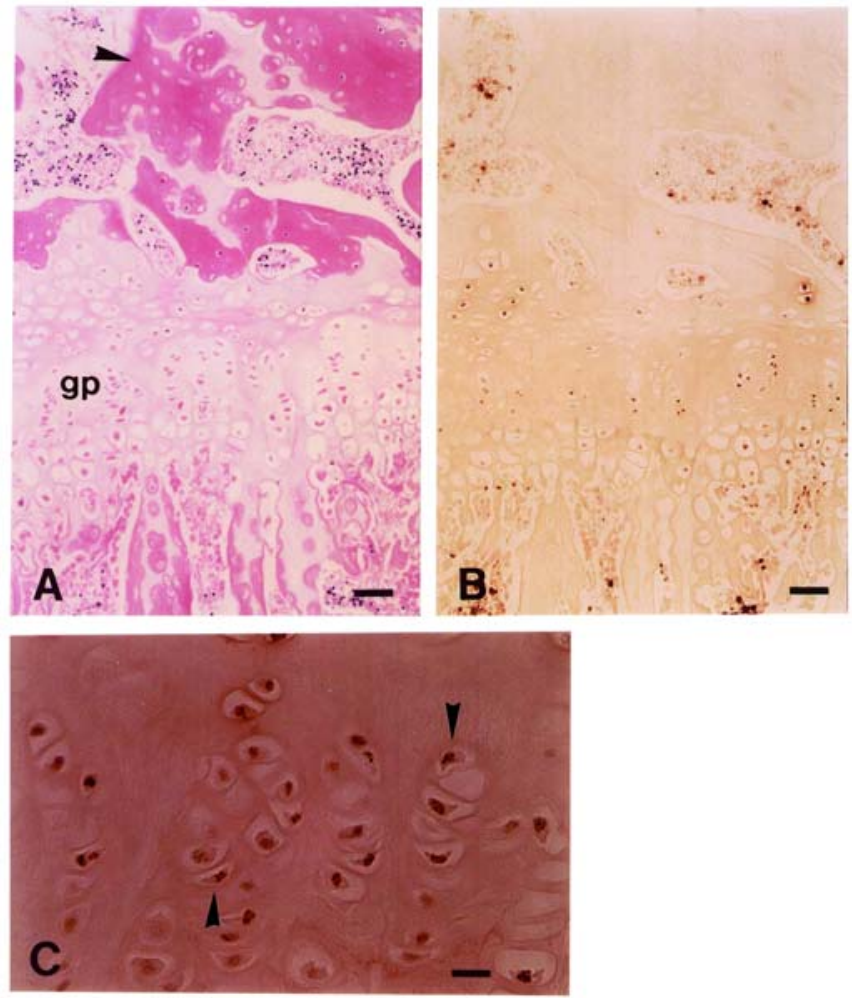

Fig. 7

Photomicrographs of the histological changes and the TUNEL reaction in a longitudinal section of the femoral head after 96 hours of ischaemia. Figure 7A - The growth plate showing empty osteocytic lacunae (arrowhead) (gp, growth plate; bar $=90 \mu \mathrm{m}$; haematoxylin and eosin). Figure 7B - The TUNEL reaction in chondrocytes and bonemarrow cells $(\mathrm{bar}=90 \mu \mathrm{m})$. Figure $7 \mathrm{C}-$ The TUNEL reaction showing apoptotic bodies in chondrocytes (arrowheads) $(\mathrm{bar}=22 \mu \mathrm{m})$. 
mic impact is the key factor in the possibility of recovery. Both mechanisms of cell death, necrosis and apoptosis, have been described during ischaemia. ${ }^{9-13}$ and although the passive necrotic pathway of cell death is not reversible, the active apoptotic way is potentially preventable.

Previous studies have shown that various stress proteins are induced by hypoxic and/or ischaemic stress (e.g. glucose-regulated and heat shock proteins). ${ }^{2}$ The two distinctive stress proteins which we have examined have been reported to be markers of ischaemic stress and have an important role in the successful adaptation of astrocytes to oxygen deprivation. Expression of ORP150, a stress protein originally purified and cloned from cultured rat astrocytes subjected to hypoxia, has been observed in several types of cell subjected to oxygen deprivation. ${ }^{3,14}$ Factors responsible for ORP150 expression are also present in atherosclerotic lesions and in cancer cells infiltrating the stroma of breast tumours. ${ }^{15-18}$ ORP150 is produced more selectively in response to oxygen deprivation than $\mathrm{HO} 1$, which displays enhanced expression in response to other stimuli. $^{3-5}$ Recently, ORP150 antisense-transfected human embryonic kidney (HEK) cells were shown to display increased vulnerability to hypoxia, with loss of cell viability due to apoptosis, compared with wild-type and sensetransfected cultures. ${ }^{6}$ Therefore ORP150 has been considered to suppress hypoxia-induced apoptotic cell death. HO1 also has been considered to play a role in cytoprotective cellular mechanisms by means of the inhibition of apoptosis, ${ }^{19,20}$ but the details of these mechanisms are not clear.

In normal femora, ORP150 and HO1 mRNA was constitutively detected in proliferative chondrocytes but not in osteoblasts, osteocytes or bone-marrow cells. By contrast, TUNEL staining and apoptotic bodies were detected in terminally differentiated hypertrophic chondrocytes. Both the gene expression and TUNEL staining were exclusive. During maturation of chondrocytes, the gene expression of ORP150 and HO1 mRNA was eliminated after hypertrophy and the disappearance of these proteins may trigger apoptosis.

Three characteristic dynamic cellular and molecular events take place during the development of osteonecrosis. The first is the induction of ORP150 and HO1 mRNA expression by the ischaemic event and this was seen much earlier than the empty lacunae typical of necrotic bone. These mRNAs began to express in osteoblasts and osteocytes after three hours of ischaemia, and also in proliferative chondrocytes. No expression was detected in osteoblasts and osteocytes after 12 hours of ischaemia, but in proliferative chondrocytes expression was still detected at 12 hours but not at 24 hours. By contrast, empty lacunae were detectable histologically after 96 hours of ischaemia. These results suggest that ORP150 or HO1 may be early and sensitive novel markers for ischaemic osteonecrosis.

The second event during ischaemic osteonecrosis is a cell-death mechanism based on apoptosis including fragmentation of DNA and the presence of apoptotic bodies in osteocytes, chondrocytes and bone-marrow cells. A TUNEL-positive reaction and apoptotic bodies began to be detected in osteocytes and bone-marrow cells after 12 hours of ischaemia, and in chondrocytes after 24 hours. These results support the contention that various cells in bone tissue undergo programmed cell death/apoptosis during ischaemic osteonecrosis, and that chondrocytes are programmed to withstand hypoxic and ischaemic stress compared with more vulnerable osteoblasts and bone-marrow cells among cell populations in bone tissue. The term osteonecrosis may be misleading, since cells were shown to undergo apoptosis, but it has not been demonstrated that the bone cells die by necrosis. Indeed, the cell swelling and tissue inflammation which characterise necrosis in soft tissues do not occur. ${ }^{21,22}$

The last event to be mentioned is the replacement of the ORP150 and HO1 mRNA signal with the TUNEL-positive reaction during development of osteonecrosis. This was detected in osteoblasts, osteocytes and chondrocytes. No TUNEL reaction had been found in any cell while ORP150 and HO1 mRNA signals were present, and the TUNELpositive reaction followed the cessation of expression of these genes. After the initial ischaemic event, the expression of ORP150 and HO1 mRNA, the TUNEL-positive reaction and empty lacunae were found sequentially. Study of these can clarify the stage of the tissue during the development of osteonecrosis. All of our results are consistent with previous observations on ORP150 antisensetransfected HEK cells ${ }^{6}$ and lend considerable strength to the hypothesis that ORP150 and/or HO1 have the capacity to suppress hypoxia-induced apoptotic cell death. ORP150 and/or HO1 are suggested to be potential preventative tools for osteonecrosis, and the prolonged expression of their mRNA by gene transfer may prevent apoptosis during ischaemic osteonecrosis.

Although it does not fully simulate clinical osteonecrosis in man, our rat model has provided new insight into the molecular mechanism of the response of bone tissue to ischaemia. Idiopathic and traumatic osteonecrosis of the femoral head in man will be subject for further study.

We thank Mr A. Fukuyama and Mr K. Morihana for their help in preparing the histological sections.

This work was supported in part by Grant in Aid for Scientific Research from the Ministry of Education, Science and Culture of Japan No. 10671360 .

No benefits in any form have been received or will be received from a commnerical party related directly or indirectly to the subject of this article.

\section{$\underline{\text { References }}$}

1. Arlet J. Nontraumatic avascular necrosis of the femoral head: past, present and future. Clin Orthop 1992;277:12-21.

2. Wilson RE, Sutherland RM. Enhanced synthesis of specific proteins, RNA and DNA caused by hypoxia and reoxygenation. Int J Radiat Oncol Biol Phys 1989;16:57-61. 
3. Kuwabara K, Matsumoto M, Ikeda J, et al. Purification and characterization of a novel stress protein, the 150-kDa oxygen-regulated protein (ORP150), from cultured rat astrocytes and its expression in ischemic mouse brain. J Biol Chem 1996;271:5025-32.

4. Maines MD, Ewing JF. Stress response of the rat testis: in situ hybridization and immunohistochemical analysis of heme oxygenase1 (HSP32) induction by hyperthermia. Biol Reprod 1996;54:1070-9.

5. Choi AM, Alam J. Heme oxygenase-1: function, regulation and implication of a novel stress-inducible protein in oxidant-induced lung injury. Am J Respir Cell Mol Bil 1996;15:9-19.

6. Ozawa K, Kuwabara K, Tamatani M, et al. 150-kDa oxygenregulated protein (ORP150) suppresses hypoxia-induced apoptotic cell death. J Biol Chem 1999;274:6397-404.

7. Nomura S, Hirakawa K, Nagoshi J, et al. Method for detecting the expression of bone matrix protein by in situ hybridization using decalcified mineralised tissue. Acta Histochem Cytochem 1993;26:303-9.

8. Blin N, Stafford DW. A general method for isolation of high molecular weight DNA from eukaryotes. Nucleic Acids Res 1976;3:2303-8.

9. Burke TJ, Schrier RW. Pathophysiology of cell ischemia. In: Schrier RW, Gottschalk C, eds. Diseases of the kidney. Vol. 2. Boston: Little Brown Company, 1993:1257-86.

10. Weinberg IM. The biology of ischemic renal injury. Kidney Int 1991;39:476-500.

11. Hagar H, Ueda N, Shah SV. Endonuclease induced DNA damage and cell death in chemical hypoxic injury to LLC-PKI cells. Kidney Int 1996;49:355-61.

12. Hagar H, Ueda $\mathbf{N}$, Shah SV. Role of reactive oxygen metabolites in DNA damage and cell death in chemical hypoxic injury to LLC-PKI cells. Am J Physiol 1996;271:209-15.
13. Beeri R, Symon Z, Brezis M, et al. Rapid DNA fragmentation from hypoxia along the thick ascending limb of rat kidneys. Kidney Int 1995;47:1806-10.

14. Ikeda J, Kaneda S, Kuwabara K, et al. Cloning and expression of cDNA encoding the human $150 \mathrm{kDa}$ oxygen-regulated protein, ORP150. Biochem Biophys Res Commun 1997;230:94-9.

15. Knighton DR, Hunt TK, Scheuenstuhl H, et al. Oxygen tension regulates the expression of angiogenesis factor by macrophages. Science 1983;221:1283-5.

16. Kuwabara K, Ogawa S, Matsumoto $M$, et al. Hypoxia-mediated induction of acidic/basic fibroblast growth factor and platelet-derived growth factor in mononuclear phagocytes stimulates growth of hypoxic endothelial cells. Proc Natl Acad Sci USA 1995;92:4606-10.

17. Tsukamoto Y, Kuwabara K, Hirota S, et al. $150-\mathrm{kD}$ oxygenregulated protein is expressed in human atherosclerotic plaques and allows mononuclear phagocytes to withstand cellular stress on exposure to hypoxia and modified low density lipoprotein. J Clin Invest 1996;98:1930-41.

18. Tsukamoto Y, Kuwabara K, Hirota S, et al. Expression of the 150 -kd oxygen-regulated protein in human breast cancer. Lab Invest 1998;78:699-706.

19. Brune B, von Knethen A, Sandau KB. Nitric oxide and its role in apoptosis. Eur J Pharmacol 1998;351:261-72.

20. Otterbein LE, Kolls JK, Mantell LL, et al. Exogenous administration of heme oxygenase-1 by gene transfer provides protection against hyperoxia-induced lung injury. J Clin Invest 1999;103:1047-54.

21. Mankin HJ. Nontraumatic necrosis of bone (osteonecrosis). $N$ Engl J Med 1992;326:1473-9.

22. Ficat RP. Idiopathic necrosis of the femoral head. J Bone Joint Surg [Br] 1985;67-B:3-9. 